\title{
The Present State of the Theory of Natural Selection
}

$\mathrm{O}^{\mathrm{N}}$ May 14, the Royal Society held a discussion on "The Present State of the Theory of Natural Selection". Prof. D. M. S. Watson, opening the discussion, emphasised the lack of evidence that structural features are adaptive. In the brachiopod Rhynchonella, specimens with the inhalent and exhalent apertures completely abnormal occur abundantly in a random collection of large numbers, of different sizes, from one locality: the abnormality has not been selected out. Much more experimental proof of a selective death-rate is needed. While a change may take place independently on parallel lines, the rate may differ; there is a compensatory principle as in the evolution of the horse, in which an advanced rate of change in the teeth is associated with a backwardness in the case of the feet. The same adaptive structure may arise independently in nearly allied animals, or the same ends may be served by different adaptations in other groups. Perissodactyls show a dental peculiarity, increasing the grinding surfaces, which artiodactyls never do ; what it is that prevents them from doing so, is a clue to evolution. Such types of evolution, common to great groups, have nothing to do with the evolution of specific characters. Do ordinary species differ from each other by adaptive characters? Quantitative results are of the utmost importance, yet they are not fortheoming.

Prof. N. Timoféeff-Ressovsky produced important figures dealing with relative viability in races of Drosophila. A state of over-crowding in a culture of the flies under optimum conditions can be produced by the introduction of others, leading to the death of a certain number. The percentage of viability of various cultures thus over-crowded shows that certain races will survive better than others at raised or lower temperatures. Although races from different areas may show no morphological differences, their viabilities at different temperatures agree with the climatic conditions of the areas they occupy. The populations of eastern Europe in a typical continental climate have higher viability at temperatures beyond normal in either direction than the north European race, which endures increased cold better than the Mediterranean race ; this, on the other hand, endures increased heat alone better than the former. There is evidence that there do exist in the wild population sufficient allelomorphs to provide material for selection for climate, and that each of the mutants has its own specific relative viability dependent upon combination with other genes; small mutations affecting viability occur at least twice as frequently as lethals.

The differential elimination of genotypes of low viability was discussed by Prof. R. A. Fisher in the case of four species of grass locusts. There was unmistakable evidence of this, by deaths between the time of formation of the zygote and the time of capture, or between the time of capture and the time of formation of the next generation. In one case, not even complete sterility would explain the disparities observed ; there must have been differential elimination of one genotype of low viability.

Prof. E. J. Salisbury urged that plants have much greater tolerance to supposedly lethal conditions than is thought to be the case; elimination is largely among juveniles. Survival is determined by reaction to competition for space; wild communities of an open character contain many microspecies. Allied microspecies grown in dense cultures show great mortality ; a denser culture of a single than of mixed microspecies can be grown. Despite Darwin's axiom that competition is greatest between individuals of the same species, if they have no different competitive equipment, enormous numbers can live together without mortality.

The view that natural selection reduces poly. morphism and favours distinct species in different environments was expressed by Dr. W. B. Turrill, who cited many cases of close adaptation to environ. ment in plant species growing in contiguous areas, differing by well-marked characters yet each not spreading from its own area. The colonisation of a new area may be aided by a difference in habit, and Dr. T. J. Jenkin ascribed the difference between the annual rye-grass occurring in South Europe and the northern perennial species to an original divergence of habit which favoured different forms in different climatic environment. Such differences were not shown in morphology; the grasses are all diploids, and can be intercrossed without any noticeable change except a disturbance of meiosis.

Close adaptations were stated by Capt. Cyril Diver to be limitations; many species do not show them, and they may even be disadvantageous. Wide variability goes with adaptability; more common species are more variable than the less common, so that a successful species may be presumed to preserve wide adaptability. If close adaptations are a measure of the working of natural selection, wide adaptability, being incompatible with them, can scarcely be attri. buted to natural selection. The important thing for a species is to be tolerant and preserve a wide adapt. ability, and not close adaptations. This is best effected if there is no selective pressure to limit these adaptations; a condition of selective pressure causing close adaptation suggests that a species is losing rather than gaining ground.

There is difficulty in believing that adaptation is due to natural selection in cases where a new form is found living under the same conditions as its parent form. This point was also emphasised by Prof. R. Ruggles Gates; there is little evidence that natural selection is aiding the spread of new forms. Thus an apetalous form of Saxifraga virgin. iensis, having started in Manhattan, has become abundant in Massachusetts ; a teratological form of Drosera spreading vegetatively has occupied an area of half an acre to the exclusion of the type form. The size of a population is of extreme importance; much depends upon whether all individuals can meet to interbreed, which is unlikely in widely scattered species. There is an effective range of interbreeding which will be less than the total range. Even migratory birds return to breed at the same spot, but plants have their effectiveness increased by necessary visits of pollinating insects or of wind. Therefore an element of chance may affect spread.

Dr. W. B. Turrill pointed out in his contribution that large changes of environment, such as is produced by man's deforestation, afford opportunities 
for establishment of a new population, and that migration might be of less importance than changes in situ.

Theories of evolution were divided by Prof. R. A. Fisher into two kinds: those that explain adaptations, and those that fail to account for them. The development of the living organism must account for adaptations, otherwise (1) species must arise by an inner urge causing a progressive predetermined course undeterred by differences of birth or death, (2) the environment must govern the course of change. The "mutation theory, once popular among geneticists", omits the consideration of adaptations, and fails to explain the functions of organisms or their parts.

Dr. R. N. Salaman, in a few words at the end of the discussion, showed that a mutation might arise which only in certain cireumstances might be of direct value to a species. A Solanum from Mexico, a country free from wart disease, has in Great Britain de. veloped a recessive, bred true, which is immune to wart: a character that is useless in its native country but of the utmost value for England.

The value of protective adaptations in mimicry among arthropods was stressed by Prof. G. D. Hale Carpenter. Mimetic effects are produced in a variety of ways all serving the same end; a characteristie product of natural selection. Polymorphism in the case of mimicry is favoured by natural selection, contrary to the views of Dr. Turrill. A species gains by resemblance to different models or many kinds of inanimate objects, as this increases the work the enemy has to do in finding food. Habit plays an important part; conspicuovs species furnished with unpleasant qualities have habits displaying them; mimies have habits enhancing the likeness, but often abandoned in real danger. The fact that mimicry deceives the artist but not the anatomist can only be explained by natural selection. The correspondence between models and mimicry in precise distribution is of utmost importance.

The question of numbers was discussed by Prof. J. B. S. Haldane. We are not in a position to detect evolutionary changes in a population at our disposal. Palæontologists agree that at least 20,000 generations are required to produce a recognisable change. If a gene is increased by 0.01 per cent, its frequency will increase in a random population from 0.01 to 99 per cent in 23,400 generations; but it would need a population of four millions to reveal it. This point had been also considered by Prof. Ruggles Gates, who said that in quite small populations the conditions would scarcely differ from ehance survivals.

Prof. Haldane also said that new genes must be such as to decrease fitness, for if not they would already have spread through a population. The primary role of natural selection is to stabilise a species. Evolutionary changes are only to be expected as a result of drastic changes in environment; there is rarely direct evidence of selection. But such evidence exists in man, in the case of resistance to a disease : the progressive diminution of tuberculosis is due to natural selection developing immunity.

Parallel mutation was discussed by Prof. Ruggles Gates : it is so abundant, causing parallel evolution, that we must look on phylogeny not as like the usual Darwinian tree, but as the root system of a fig-tree which sends down parallel roots, descending and interlacing.

The facts of mimicry in insects, however, according to Prof. Hale Carpenter, cannot be explained by parallel mutation. The results in mimics and models are not the same, and similar effects are produced in different ways. One of a series of forms of a polymorphic mimetic species, of which the majority mimic species of one subfamily, may mimic a species of quite a different subfamily, and yet this form is linked with the others by intermediates.

\section{The Mysterious Number 137}

$\mathrm{I}^{\mathrm{T}}$ is a remarkable fact, first made prominent by Sommerfeld's discussion of the fine structure of the hydrogen spectrum, that from three physical constants, $h$ (Planck's constant), $c$ (the velocity of light in vacuo) and $e$ (the charge on an electron), a dimensionless pure number can be formed, which usually occurs in the form $h c / 2 \pi e^{2}$, with the numerical value 137 , or more accurately $137 \cdot 2$.

Dr. Max Born, in a lecture delivered to the South Indian Science Association at Bangalore on November 9, 1935 (Proc. Indian Acad. Sci., 2, 533; 1935), declared that the explanation of this number must be the central problem of natural philosophy. Its existence can be ascribed to the fact that there are two different 'natural' units of length, a larger one $\lambda_{0}=h / m c$ (the so-called Compton wave-length) taken from quantum theory, and a smaller one $a_{0}=e^{2} / m c^{2}$ (the so-called radius of the electron). Their ratio is $2 \pi$ times the mysterious number. After pointing out the great importance of this number in atomic physics, Dr. Born criticised the existing explanations of it.

Sir Arthur Eddington considers that it is associated with the number of degrees of freedom of a pair of electrons, and obtains the value 137. Dr. Born rejects this view, and seeks for an alternative explanation based on the new Born-Infeld-Pryce unitary field theory, which considers matter and field as one and the same. It involves a very large constant called 'the absolute field', which is the magnitude of the field in the centre of the electron. It is suggested that the number 137 is related to the neutralisation frequency of oscillation of a pair of electrons, one positive and the other negative (produced by light quanta passing the field of a nucleus), which approach and finally neutralise each other, emitting light quanta. Apparently the details of the calculation have not been worked out on Born's own theory, but by working on a somewhat similar theory Euler and Kockel (two pupils of Heisenberg) obtain the value $82 \cdot 4$. This differs considerably from the value $137 \cdot 2$, but Dr. Born considers the discrepancy not discouraging in view of the arbitrary assumptions made in the theory.

Dr. Born also uses the new field theory to explain the ratio of the proton and the electron, and obtains the number 2340 . The experimental value is $1846 \cdot 6$, and the theory of Sir Arthur Eddington gives the value $1847 \cdot 6$ (Proc. Roy. Soc., A, 134, 524 ; 1931). 